\title{
Gallbladder Paraganglioma: Computed Tomography and Magnetic Resonance Imaging Findings
}

\author{
Mustafa Koplay', Mesut Sivri', Hüsnü Alptekin², Hasan Erdoğan', \\ Alaaddin Nayman ${ }^{1}$ \\ ${ }^{1}$ Department of Radiology, Medical Faculty, Selcuk University, Konya, Turkey; \\ ${ }^{2}$ Department of General Surgery, Medical Faculty, Selcuk University, Konya, Turkey \\ Received July 6, 2014; Accepted November 18, 2014.
}

Key words: Gallbladder - Paraganglioma - CT - MRI

Abstract: Paragangliomas are benign tumors that can be association with sympathetic and parasympathetic nerves. More rarely, they can be located in the gallbladder. In this paper, we describe the appearance of an unusual case of paraganglioma in the gallbladder.

Mailing Address: Mustafa Koplay, MD., Department of Radiology, Medical Faculty, Selcuk University, Central Campus, 42075, Konya, Turkey; Phone: +90 33222 43800-44930; e-mail: koplaymustafa@hotmail.com 


\section{Introduction}

Paraganglioma is benign tumor that develops from neural crest primitive cells called paraganglia. It is extremely rare to see it in the gallbladder. In the literature, only few cases have been reported (Cho et al., 2001). In this report we present an extremely rare case of gallbladder paraganglioma and its imaging findings.

\section{Case report}

A 57-year-old woman referred to our hospital with right upper quadrant abdominal pain for fifteen days. There was no liver disease, hypertension, smoking, alcohol ingestion, or jaundice in her medical history. Physical examination was without major findings and results of laboratory test were normal. Abdominal ultrasound showed a hypoechoic solid mass at the neck of gallbladder in size $17 \times 25 \mathrm{~mm}$. Computed tomography (CT) and magnetic resonance imaging (MRI) was performed for the differential diagnosis. Contrast-enhanced CT scans showed a well-marginated soft tissue mass with peripheral enhancement and low attenuation of central areas (Figure 1). On T1 and T2-weighted images, the lesion was hypointense and hyperintense respectively and there was intense homogeneous enhancement in contrast-enhanced images. On T2-weighted images the appearance of high-signal and low-signal regions resulted in a "salt and pepper" heterogeneity (Figure 2). Pre-surgery biopsy wasn't performed because patient had not accepted biopsy. The mass was removed laparoscopically. At laparoscopic operation a well-circumscribed mass of $2.5 \mathrm{~cm}$ diameter was seen in the distal wall of the gallbladder. In pathological examination, the mass located into the serosa
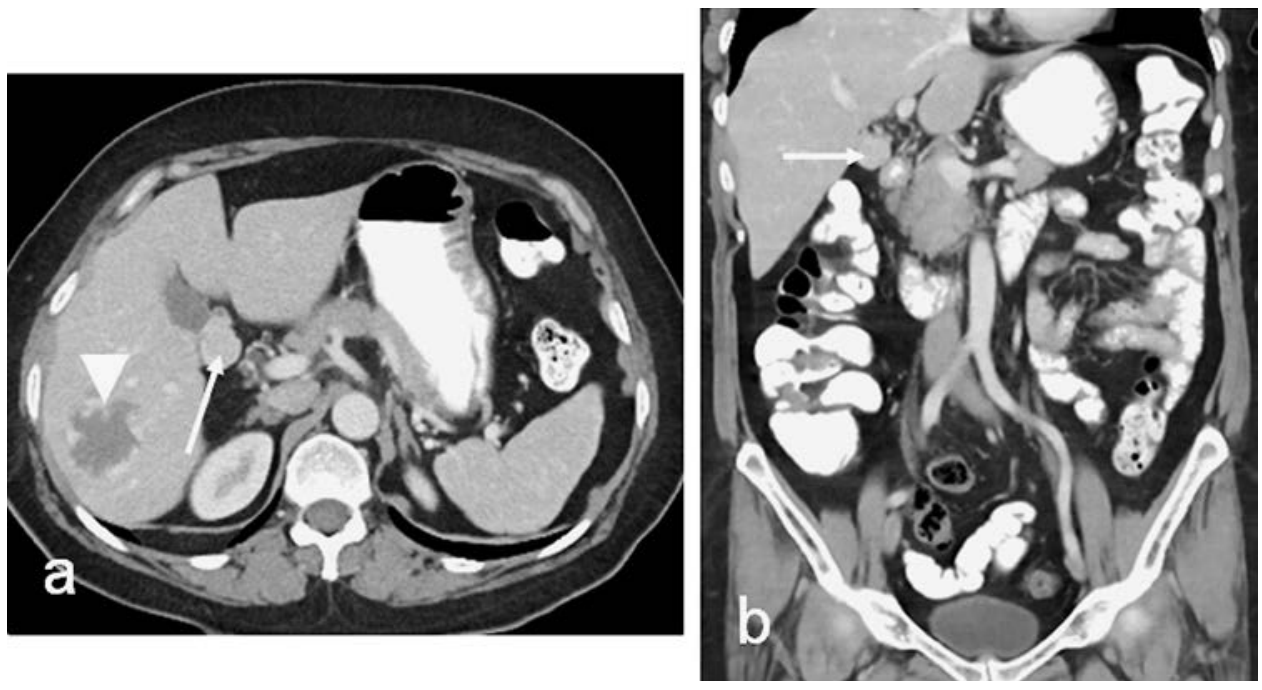

Figure 1 - Contrast-enhanced axial (a) and coronal (b) CT images show a well-marginated soft tissue mass with peripheral enhancement and low attenuation of central areas (arrows). In addition, axial CT image shows a hemangioma in liver right lobe (arrowhead). 


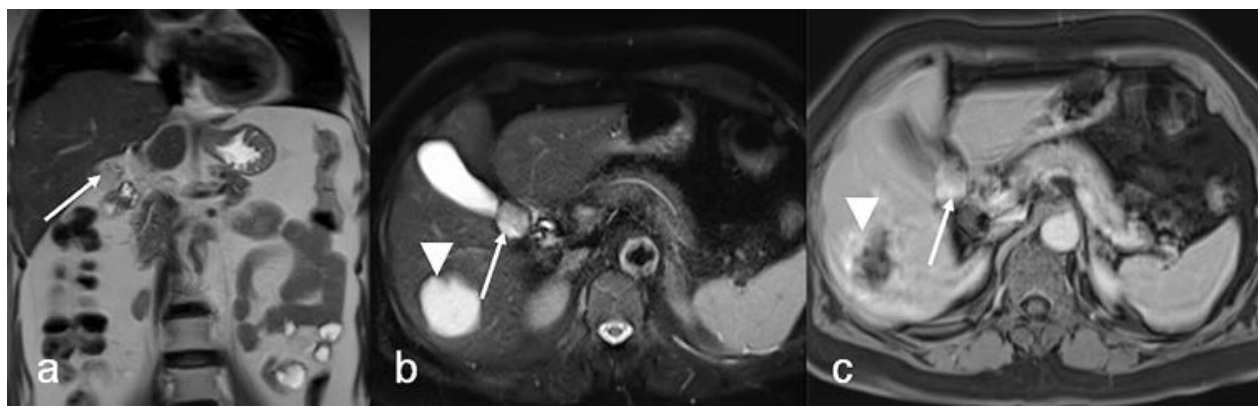

Figure 2 - Coronal T2-weighted (a) and axial T2-weighted fat-saturated (b) images show as mild hyperintense and "salt and pepper" appearance in lesion (arrow). Axial T1-weighted fat-saturated contrast enhanced image (c) shows intense enhancement; arrow - paraganglioma; arrowhead - hemangioma.

and muscular layer and did not exceed the serosa. Immunohistochemical staining revealed tumor cells which were highly positive for synaptophysin, and less positive for vimentine and chromogranin. S100 was positive in sustentacular cells. The case was reported as a paraganglioma.

\section{Discussion}

Paragangliomas are predominantly benign tumors which arise from cells in the paraganglia. In about $10 \%$ of patients it can manifest a malignant transformation. This tumor can develop in association with sympathetic and parasympathetic nerves such as the carotid body, jugular foramen, vagus nerve, organ of Zuckerkandl, mediastinum and retroperitoneum. More rarely, it can occur in orbita, cauda equina, duodenum, urinary bladder, prostate and thyroid gland (Kliewer and Cochran, 1989). It is extremely rare to see it in the gallbladder. The first case was reported by Miller in 1972 as an incidental finding (Miller et al., 1972). Until today very little cases have been reported. In general, tumors are detected incidentally or secondary to their complications. In these cases, gallbladder paragangliomas were predominantly seen in females and in the fifth to sixth decade. The size was usually small ranged from 1.3 to $5 \mathrm{~cm}$.

The imaging features of paraganglia are usually similar as an homogeneous or heterogeneous increased enhancing soft-tissue mass at CT because of their hypervasculity. On MRI, they have low or intermediate signal intensity on T1weighted images and high signal intensity on T2-weighted images. There are high and low-signal regions of the tumor described as "salt and pepper" appearance on T2-weighted images because of slow flow or hemorrhage and signal voids of tumor vessels (Lee et al., 2006). Metaiodobenzylguanidine (MIBG) scintigraphy is a functional imaging technique. It is a norepinephrine analogue labelled with 123 or $131 \mathrm{l}$ and helps to identify multiple primary tumors and metastases. Its specificity is $95-100 \%$, sensitivity is $85 \%$, when combined with CT and MRI, the sensitivity 
is increasing (Shapiro et al., 1995). Positron emission tomography (PET) has been increasingly used in diagnosis.

In conclusion, we present a rare case of paraganglioma that occurred in gallbladder. Because of gallbladder paraganglioma is rare; it doesn't come to mind in the differential diagnosis of gallbladder masses. It is important to know the radiological manifestation of this rare unusual tumor for diagnosis and these findings may be helpful the radiologists to think the possibility of paraganglioma during differential diagnosis.

\section{References}

Cho, Y. U., Kim, J.Y., Choi, S. K., Hur, Y. S., Lee, K.Y., Kim, S. J., Ahn, S. I., Hong, K. C., Woo, Z. H., Han, J.Y., Shin, S. H (2001) A case of hemorrhagic gallbladder paraganglioma causing acute cholecystitis. Yonsei Med.J. 42, 352-356.

Kliewer, K. E., Cochran, A. J. (1989) A review of the histology, ultrastructure, immunohistology, and molecular biology of extra-adrenal paragangliomas. Arch. Pathol. Lab. Med. 113, 1209-1218.

Lee, K.Y., Oh, Y.W., Noh, H. J., Lee, Y. J., Yong, H. S., Kang, E.Y., Kim, K. A., Lee, N. J. (2006) Extraadrenal paragangliomas of the body: imaging features. AJR Am. J. Roentgenol. 187, 492-504.

Miller, T. A., Weber, T. R., Appelman, H. D. (1972) Paraganglioma of gallbladder. Arch. Surg. 105, 637-639.

Shapiro, B., Sisson, J. C., Shulkin, B. L., Gross, M. D., Zempel, S. (1995) The current status of metaiodobenzylguanidine and related agents for the diagnosis of neuro-endocrine tumors. Q. J. Nucl. Med.

39, 3-8. 